\title{
Erratum to: Identifying decreased peristalsis of abnormal small bowel segments in Crohn's disease using cine MR enterography: the frozen bowel sign
}

\author{
Flavius F. Guglielmo, ${ }^{1}$ Donald G. Mitchell, ${ }^{1}$ Patrick L. O'Kane,${ }^{1}$ Sandeep P. Deshmukh, ${ }^{1}$ \\ Christopher G. Roth, ${ }^{2}$ Ilene Burach, ${ }^{3}$ Aaron Burns, ${ }^{4}$ Susan Dulka, ${ }^{5}$ Laurence Parker ${ }^{1}$ \\ ${ }^{1}$ Department of Radiology, Thomas Jefferson University, 132 South 10th Street, Philadelphia, PA 19107, USA \\ ${ }^{2}$ Department of Radiology, Methodist Hospital, Thomas Jefferson University, 2301 South Broad Street, Philadelphia, PA 19148, \\ USA \\ ${ }^{3}$ Shore Medical Center, 100 Medical Center Way, Somers Point, NJ 08244, USA \\ ${ }^{4}$ Our Lady of Lourdes Medical Center, 1600 Haddon Avenue, Camden, NJ 08103, USA \\ ${ }^{5}$ Radiology Center, M.C. 34-46, 1000 E Mountain Blvd., Wilkes-Barre, PA 18711, USA
}

\begin{abstract}
Purpose: The purpose of this study was to evaluate whether affected bowel in Crohn's disease patients can be identified by observing decreased peristalsis (frozen bowel sign) using cine balanced steady-state free precession (cine BSSFP) images.

Materials and methods: 5 radiologists independently reviewed cine BSSFP sequences from randomized MR Enterography (MRE) exams for 30 normal and 30 Crohn's disease patients, graded overall small bowel peristalsis from slowest to fastest, and graded peristalsis for the most abnormal small bowel segment. Sensitivity and specificity of the frozen bowel sign for diagnosing Crohn's disease were calculated. T tests of the peristalsis difference between abnormal segments and overall small bowel were conducted.

Results: For 5 readers, the sensitivity and specificity of cine BSSFP of the frozen bowel sign for diagnosing Crohn's disease ranged from $70 \%$ to $100 \%$ and $87 \%$ to $100 \%$, respectively. There were significant differences in
\end{abstract}

Electronic supplementary material: The online version of this article (doi:10.1007/s00261-014-0293-8) contains supplementary material, which is available to authorized users.

The online version of the original article can be found under doi: 10.1007/s00261-014-0258-y.

Correspondence to: Flavius F. Guglielmo; email: flavius.guglielmo@, jefferson.edu peristalsis between abnormal small bowel segments and the overall small bowel for Crohn's patients, but not in the overall small bowel between normal-MRE patients and Crohn's disease patients.

Conclusion: Abnormal Crohn's small bowel segments have significantly decreased peristalsis compared to normal small bowel, which can be identified using cine BSSFP sequences as the frozen bowel sign.

Key words: MR enterography - Small bowelPeristalsis-Motility-Cine MRI-Crohn's disease

\section{Erratum to: Abdom Imaging DOI 10.1007/s00261-014-0258-y}

The original publication did not include the images pertaining to the supplementary video files. This has been corrected in this article.

Using MR enterography (MRE) to diagnose inflamed small bowel segments in patients with Crohn's disease requires a multiparametric approach, evaluating imaging features from several different pulse sequences. The most commonly used imaging features are well established in the literature for both CT scan [1-4] and MRI [5-15]. These include bowel wall thickening, bowel wall edema, perienteric edema and fluid, and mucosal, transmural, and possibly serosal hyperenhancement after intravenous contrast or gadolinium administration. With MRE, these 
well-known imaging features are evaluated using a combination of unenhanced and post-contrast T1-weighted images, usually with fat suppression, T2-weighted images with and without fat suppression, and more recently diffusion-weighted images [16-19]. However, there are times when these sequences are not completely diagnostic for inflammatory bowel disease, and having additional imaging criteria would be useful.

Small bowel peristalsis evaluation is another method to determine if the small bowel is inflamed, thus making functional evaluation an additional imaging parameter. First established with fluoroscopy, small bowel peristalsis can be evaluated with MRE using a cine balanced steady-state free precession series (cine BSSFP) [20]. In patients with known Crohn's disease, inflamed small bowel segments can have motility changes which usually manifest as decreased or absent peristalsis compared to normal small bowel segments [21-32].

The utility of small bowel peristalsis evaluation as an additional parameter to diagnose inflamed small bowel segments is well established in prior publications when there is a known diagnosis of Crohn's disease [27-31]. However, many times in clinical practice the diagnosis of Crohn's disease is not known, and MRE is performed to determine if inflammatory bowel disease is present. In this case, having a sequence which can be used to detect inflamed bowel segments without a priori knowledge of underlying inflammatory bowel disease is valuable.

Thus, the purpose of our study was to determine if abnormal small bowel segments in Crohn's disease patients can be identified by observing decreased peristalsis using the cine BSSFP series alone, without knowing if a patient has inflammatory bowel disease and without referring to other pulse sequences. We also introduce an imaging sign which describes this decreased peristalsis called the "frozen bowel sign."

\section{Materials and methods}

\section{Patients}

Institutional Review Board approval was obtained for this retrospective study prior to evaluating archived imaging studies and examining the electronic medical records. MRE exams were collected from our institution's PACS between January 1, 2008 and December 23, 2011 using the following search criteria: "MR Enterography," "Small Bowel Enterography," "MRE," "VoLumen," "Enterography," and "Crohn's Disease." This search yielded a total of 655 cases from which MRE exams were randomly selected for 60 patients $(23$ male, 37 female; age range 15-76 years; mean age 41.8 years) including 30 normal and 30 abnormal exams. All selected exams included a cine series obtained without prior administration of any antiperistaltic agent. All 30 patients with abnormal exams had a clinical diagnosis of Crohn's disease and active and/or chronic Crohn's disease diagnosed on MRE. Of these 30 patients, 12 had active bowel inflammation, 12 had active with chronic bowel inflammation, and 6 had chronic bowel inflammation on MRE. The 30 patients with normal MRE exams ("normal-MRE patients") had a variety of indications such as weight loss, abdominal pain, mesenteric ischemia, appendiceal polyp, duplication cyst, and GI bleed, but no documented Crohn's disease or inflammatory bowel disease on either the clinical history or MRE. Exclusion criteria included (1) MRE report stating the examination was limited by motion artifact; (2) any MRE performed without intravenous contrast; and (3) if a $0.1 \%$ weight per volume barium sulfate suspension (VoLumen; Bracco Diagnostics, Princeton, NJ) was not administered or it was unclear if this was administered based on the radiology report and/or documentation by the MR technologist.

\section{Reference standard}

Of the 30 patients with abnormal MRE exams, Crohn's disease was confirmed with histopathology specimens in 26 patients obtained by endoscopic biopsy in 22 patients and surgical resection in 4 patients. The 4 remaining patients with a clinical diagnosis of Crohn's disease had prior endoscopic biopsies confirming inflammation. Concordant pathology results within 6 months of the MRE exam were available for 13 patients and within 12 months for 16 patients.

\section{$M R$ enterography}

MRE examinations were performed on 1.5 T MR Systems (Philips Intera, Philips Medical Systems, Best, Netherlands; Philips Achieva, Philips Medical Systems, Best, Netherlands; Siemens Espree, Siemens Medical Solutions, Erlangen, Germany; or General Electric Signa HDxt, GE Medical Systems, Milwaukee, Wisconsin). Multichannel phase array coils were used for each case including a SENSE body coil (Philips Intera), SENSE XL torso coil (Philips Achieva), 8-channel body coil (GE Signa HDxt) or 2 contiguous torso coils (Siemens Espree). Patients were required to fast for $2-4 \mathrm{~h}$ before the exam. One bottle $(450 \mathrm{ml}$ each) of VoLumen was administered orally every $20 \mathrm{~min}$, beginning $1 \mathrm{~h}$ before the exam. When possible, patients were scanned in the prone position $(N=51 / 60$ patients were scanned prone; $N=5 / 9$ of patients scanned supine were Crohn's disease patients). For all cases, pre-gadolinium coronal and axial single-shot heavily $\mathrm{T} 2$-weighted images $(\mathrm{TE} \sim 200)$ and post-gadolinium axial fat suppressed moderately T2-weighted images (TE 80) were obtained. Pre- and post-gadolinium 3D gradient echo (3D GRE) images were obtained using $0.1 \mathrm{mmol} / \mathrm{kg}$ of gadobenate dimeglumine (MultiHance, Milano, Italy) at a rate of $2 \mathrm{~mL} / \mathrm{s}$ using a power injector (Spectris Solaris; Medrad, 
Table 1. MRI pulse sequence parameters ranges (lowest, highest)

\begin{tabular}{|c|c|c|c|c|c|c|}
\hline & Axial 3D GRE & Coronal 3D GRE & Coronal SS T2WI & Axial SS T2WI & Axial T2 FS & CINE \\
\hline $\begin{array}{l}\text { Field of } \\
\text { view }(\mathrm{mm})\end{array}$ & 360,400 & 360,420 & 360,420 & 360,400 & 360,400 & 360,420 \\
\hline Matrix & $\begin{array}{l}256 \times 114 \\
256 \times 224\end{array}$ & $\begin{array}{l}256 \times 128 \\
320 \times 224\end{array}$ & $\begin{array}{l}256 \times 154 \\
320 \times 208\end{array}$ & $\begin{array}{l}256 \times 190 \\
324 \times 259\end{array}$ & $\begin{array}{l}256 \times 206 \\
304 \times 238\end{array}$ & $\begin{array}{l}256 \times 160 \\
320 \times 240\end{array}$ \\
\hline Flip angle & 10,15 & 10,15 & 90 & 90 & 90 & 45,70 \\
\hline $\mathrm{TR}(\mathrm{ms})$ & 3,5 & 4,5 & $\infty^{\mathrm{a}}$ & $\infty^{\mathrm{a}}$ & $\infty^{\mathrm{a}}$ & $3.1,5.1$ \\
\hline $\mathrm{TE}(\mathrm{ms})$ & $1.4,2.2$ & $1.8,2.1$ & 180,200 & 200 & 77,82 & $1.5,2.3$ \\
\hline $\begin{array}{l}\text { Section } \\
\quad \text { thickness (mm) }\end{array}$ & $2,4.5$ & $2,4.5$ & 5,6 & 5,6 & 5,6 & 8 \\
\hline $\begin{array}{l}\text { Intersection } \\
\text { gap }(\mathrm{mm})\end{array}$ & $50 \%$ overlap & $50 \%$ overlap & 0,1 & 0,1 & 0,1 & 1.5 \\
\hline $\begin{array}{l}\text { NSA } \\
\text { No of phases } \\
\text { per image/\# } \\
\text { images }\end{array}$ & $0.75,1$ & $0.75,1$ & 0.6 & 0.6 & 0.6 & $\begin{array}{l}1 \\
25 / 6,25 / 20\end{array}$ \\
\hline
\end{tabular}

$3 D$ three dimensional, $B H$ breath hold, $F O V$ field of view, $F S$ fat suppression, $G R E$ gradient echo, $M R C P$ magnetic resonance cholangiopancreatography, NSA number of signal averages, $S S$ single shot, T2WI T2-weighted image, $T E$ echo time, $T R$ repetition time

a There is no TR (repetition time) in single-shot fast spin echo pulse sequences

Warrendale, PA). Pre-gadolinium and two coronal dynamic post-gadolinium (3D GRE) series followed by a coronal delayed (3D GRE) series approximately $5 \mathrm{~min}$ later were obtained. Delayed post-gadolinium axial (3D GRE) images were also obtained. Finally, a coronal cine repetitive balanced steady-state free precession series was obtained with free breathing. For cine series, 150-500 images were obtained at 6-20 locations, each containing 25 phases and a series acquisition time of approximately $4 \mathrm{~min}$. The cine temporal resolution was $1-2 \mathrm{~s}$ per image and the flip angle ranged from $45^{\circ}$ to $70^{\circ}$, depending on the MR system. The specific pulse sequence parameters are listed in Table 1 including ranges from the lowest to the highest.

\section{Image analysis}

Cine sequences with examples of 5 progressive grades of peristalsis ranging from 1 to $5 \quad(1=$ slowest and 5 = fastest) were created by the study coordinators on a digital PACS workstation (Philips iSite Version 3.5.86, Philips Healthcare, Andover, MA, USA), based on reviewing the cine sequences from study patients and numerous other non-study patients (Fig. 1). After this, 5 abdominal imaging attending radiologists with between 5 and 25 years of MRI experience (FG, 12 years; DM, 25 years; PO, 13 years; SD, 5 years; CR, 9 years) were trained by reviewing the cine series that demonstrated the 5 peristalsis grades as well as 4 abnormal MRE exams. None of the 9 training MRE exams were from the study population. Next, the 5 radiologists were asked to review in isolation the cine series from the 30 normal and 30 abnormal study cases, in random order. The readers were blinded to other images and any clinical information. Cine sequences were reviewed on a Philips iSite PACS workstation in a manner that is similar to interpreting the entire MRE study although no other sequences were reviewed. To avoid reader fatigue, there was no restriction on the amount of time required to review each cine sequence and there was no requirement to complete all 60 interpretations at one time. For each case, using only the cine sequence at a default frame rate of 6 frames per second (which was the same rate as the 9 training cases), overall small bowel peristalsis was graded from 1 to 5 . Then, the radiologist was asked to analyze the entire small bowel for an abnormal segment, which was defined as a segment with decreased peristalsis relative to the overall small bowel plus abnormal morphology such as wall thickening, fixed narrowing, or abnormal signal intensity. Since the cine pulse sequence is used for evaluating bowel motility, peristalsis was the predominant feature evaluated. If there was one or more small bowel segment with decreased peristalsis, the most abnormal segment was graded from 1 to 5 and then marked by the radiologist to indicate which segment was chosen. Finally, the level of confidence that this bowel segment was abnormal and was recorded using a scale of 1 (lowest) to 5 (highest).

\section{Statistical analysis}

Sensitivity and specificity were calculated for the identification of Crohn's by the presence or absence of an abnormal small bowel segment. This binary judgment was expanded by the 5-point confidence rating into a 10point scale: the highest confidence level + the judgment of an abnormal segment received the highest score; the lowest confidence level + the judgment of an abnormal segment a score just above the midpoint; the lowest confidence level + the judgment of no abnormal segment a score just below the midpoint; and the highest confidence level + the judgment of no abnormal 

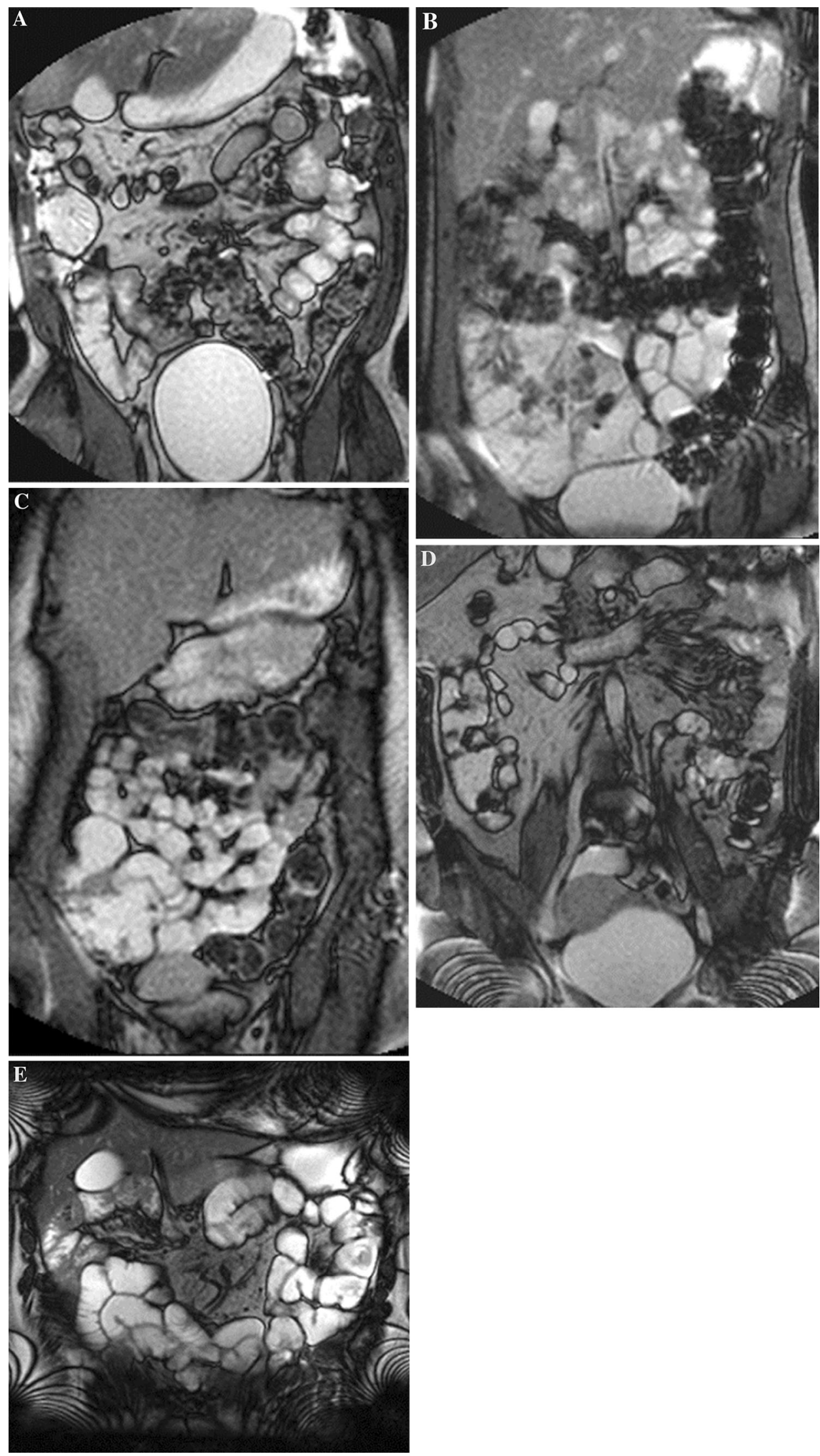

Fig. 1. Cine balanced steady state free precession (cine BSSFP) series showing the 5 progressive grades of peristalsis ranging from Peristalsis Grade 1 (slowest) to Peristalsis Grade 5 (fastest) [A (Video 1) $=$ Peristalsis Grade 1 , B $($ Video 2$)=$ Peristalsis Grade 2, C (Video 3) = Peristalsis Grade 3, D $($ Video 4$)=$ Peristalsis Grade 4, E (Video 5) = Peristalsis Grade 5]. 
segment received the lowest score. An ROC curve was calculated for this expanded score. Finally, comparisons of the level of motility, as rated on the 5-point scale, were made between the abnormal segment and remainder of the small bowel in Crohn's patients, between the abnormal segment in Crohn's patients and the whole small bowel in normal-MRE patients, and between the remainder of the small bowel in Crohn's patients and the whole small bowel in normal-MRE patients. These comparisons are not symmetrical: the first is analyzed with a paired $t$ test, the latter two with unpaired tests; and the first and third comparisons have 30 Crohn's and 30 normal patients, while the second has less than 30 Crohn's patients when a reader is inaccurate in identifying Crohn's patients. All statistical analyses were performed with SAS v.9.3 for Windows (SAS Institute, Cary, NC). The Shrout-Fleiss random set intraclass correlation (ICC) [33] was used to measure reliability of the abnormal segment and the overall peristalsis rating. It is interpreted much like Kappa.

\section{Results}

The abnormal small bowel segments included the terminal ileum in 20 patients (Figs. 2, 3, and 4), ileum in 6 patients (Fig. 5), and neoterminal ileum in 4 patients. The terminal or neoterminal ileum was arbitrarily defined as the last $10 \mathrm{~cm}$ of the ileum before the ileocecal junction or an ileocolic anastomosis, respectively [34]. Sensitivity and specificity for diagnosing Crohn's disease by identification of inflamed small bowel segments using the cine BSSFP sequences were calculated for each reader (Table 2). They were $100 \%, 100 \% ; 70 \%, 93 \%$; 97\%, 100\%; 100\%, 100\%; and 87\%, 97\%. The combined ROC curve for all 5 readers is shown in Fig. 6. The area under the curve is 0.98 . Means and standard deviations of overall small bowel peristalsis vs. an abnormal small bowel segment in Crohn's patients were calculated (Table 3). Paired $t$ tests of the peristalsis in abnormal small bowel segments in Crohn's patients vs. peristalsis in the remainder of the small bowel was significant for all five readers, with the abnormal small bowel segment lower by $2.0,1.7,1.2,1.9$, and $1.5(t=9.63-26.91$, $p<.0001)$. Means and standard deviations of overall small bowel peristalsis in normal-MRE patients and peristalsis in abnormal small bowel segments in Crohn's patients were calculated (Table 4). Independent group t tests of the peristalsis in abnormal small bowel segments in Crohn's patients with the overall small bowel peristalsis in normal-MRE patients is also significant for each reader, with the abnormal segment lower by 2.1, $1.9,1.7,2.3$ and $1.8(t=9.54$ to $17.38, p<.0001)$. Means and standard deviations of overall small bowel peristalsis in normal-MRE patients and Crohn's patients were calculated (Table 5). The difference between overall small bowel peristalsis for normal-MRE patients vs.
Crohn's disease patients was not statistically significant, except for reader $3(t=2.14, p<0.05)$. The ICCs calculated for interobserver reliability were .39 for overall small bowel peristalsis and .79 for detecting an abnormal small bowel segment.

\section{Discussion}

Our results show that using cine BSSFP sequences with a 5-point quantitative grading system, blinded to a prior history of inflammatory bowel disease and without referring to other sequences, radiologists can differentiate normal from abnormal small bowel segments, allowing Crohn's disease to be diagnosed with high sensitivity and specificity. This includes detection of abnormal small bowel segments compared to the overall small bowel in both Crohn's disease patients and patients without inflammatory bowel disease. However, we found no significant difference in overall small bowel peristalsis between patients with Crohn's disease and those without a history of inflammatory bowel disease. This latter finding differs from Bickelhaupt et al. who prospectively evaluated 13 symptomatic Crohn's disease patients and showed that small bowel peristalsis decreased in both diseased and non-diseased segments in Crohn's disease patients [29]. This discrepancy in overall small bowel peristalsis may be related to patient selection and a relatively lower degree of acuity of bowel inflammation in our patient population which included patients with active and chronic Crohn's disease.

To the best of our knowledge, this is the first study that used cine MRE to detect abnormal small bowel peristalsis to diagnose Crohn's disease when interpreting radiologists were blinded to patient history. In the study by Menys et al., the terminal ileum was evaluated in 28 patients, all of which had prior histologically proven Crohn's disease and endoscopic terminal ileal biopsy within 4 weeks of the MRE [28]. Froehlich et al. evaluated MRE studies for 40 patients with biopsy-proven Crohn's disease and clinically active Crohn's disease at the time of the MRE [31]. Girometti et al. prospectively evaluated 52 Crohn's disease patients with clinical evidence of active Crohn's disease onset or relapse [30]. Kitazume et al. evaluated MRE studies for 6 Crohn's disease patients who had longitudinal ulcers in the terminal ileum or ileum that was confirmed by colonoscopy, contrast radiology, or surgery [27]. Finally, Bickelhaupt et al. prospectively evaluated 13 patients with a known diagnosis of Crohn's disease [29]. In all prior similar cine MRE literature, the diagnosis of Crohn's disease was known without comparison to a control group. We chose our methodology to address the relatively common clinical situation of interpreting MRE exams to identify inflammatory bowel disease without a confirmed diagnosis of Crohn's disease. The fact that 5 radiologists could accurately diagnose inflamed small 

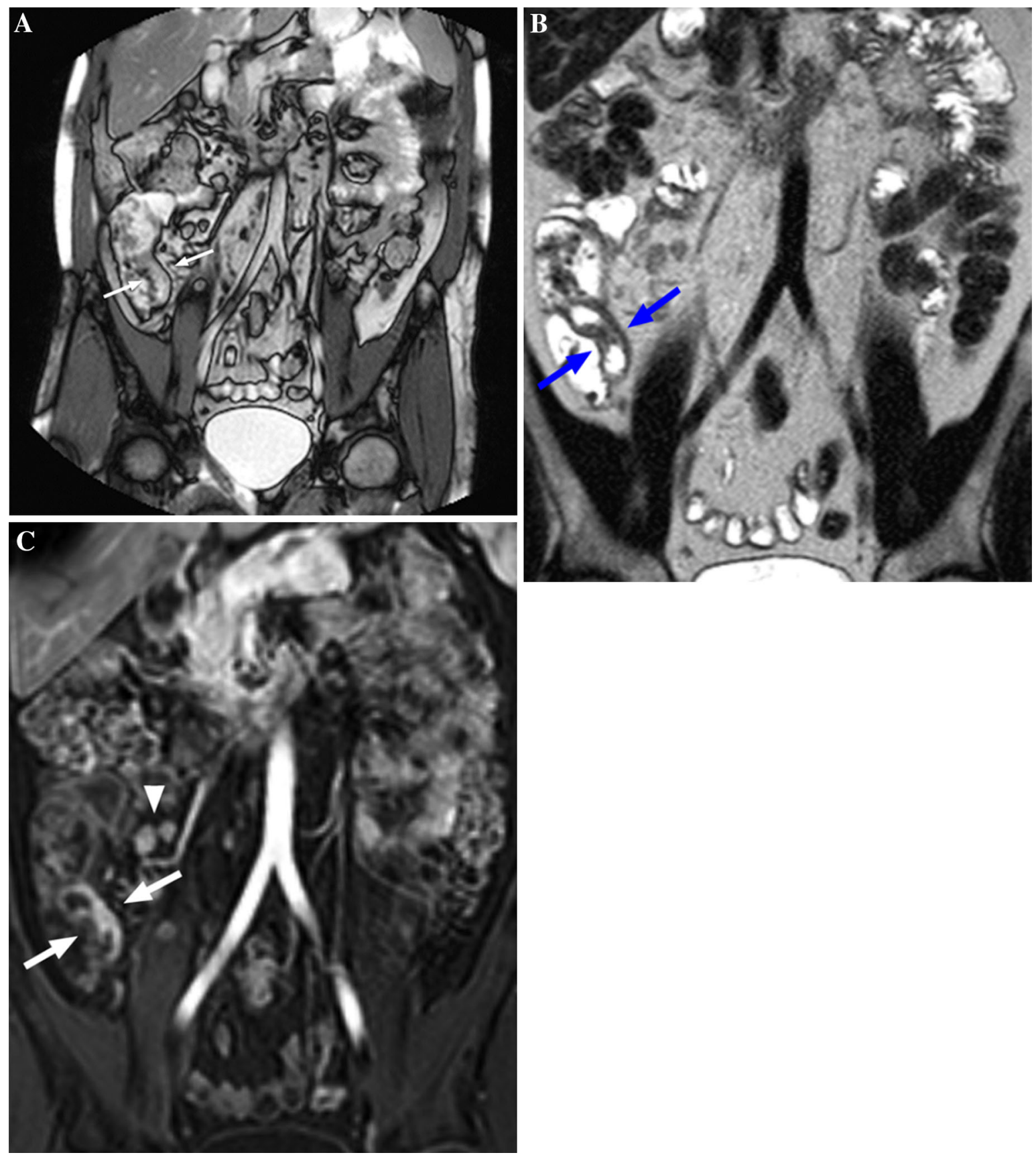

Fig. 2. Active Crohn's disease of the terminal ileum in a 33-year-old woman. A (Video 6)-Cine balanced fast field echo (BFFE) series (TR $3.2 \mathrm{~ms}$, TE $1.6 \mathrm{~ms}$, matrix $176 \times 178$, flip angle $45^{\circ}$, thickness $8 \mathrm{~mm}$ ) shows decreased peristalsis of the abnormal terminal ileum (arrow) compared to the remainder of the small bowel. B Coronal (TR $\infty$, TE $200 \mathrm{~ms}$, matrix $308 \times 268$, thickness $6 \mathrm{~mm}$ ) T2-weighted single-shot fast spin echo (FSE) image showing mild terminal

ileum wall thickening and wall edema (blue arrows). C 3-D dynamic post-contrast coronal T1W high-resolution isotropic volume examination (THRIVE) image (TR $4.1 \mathrm{~ms}$, TE $2 \mathrm{~ms}$, matrix $168 \times 167$, thickness $5 \mathrm{~mm}$ ) shows increased late arterial transmural enhancement predominantly in the mesenteric wall (arrows) with enlarged adjacent lymph nodes (arrowhead) consistent with active Crohn's disease.

bowel segments without a priori knowledge of the diagnosis of Crohn's disease further supports the diagnostic value of cine BSSFP sequences for this indication.

Many centers use a spasmolytic agent to decrease motion-induced bowel blur in an effort to improve the

image quality of 3D gradient echo (3D GRE) series, which can take up to $20 \mathrm{~s}$ to acquire. In one European study, 92\% of academic radiologists surveyed used a spasmolytic agent, most commonly hyoscine butylbromide $\left(\right.$ Buscopan $^{\circledR}$, Boehringer-Ingelheim, Ingelheim, 

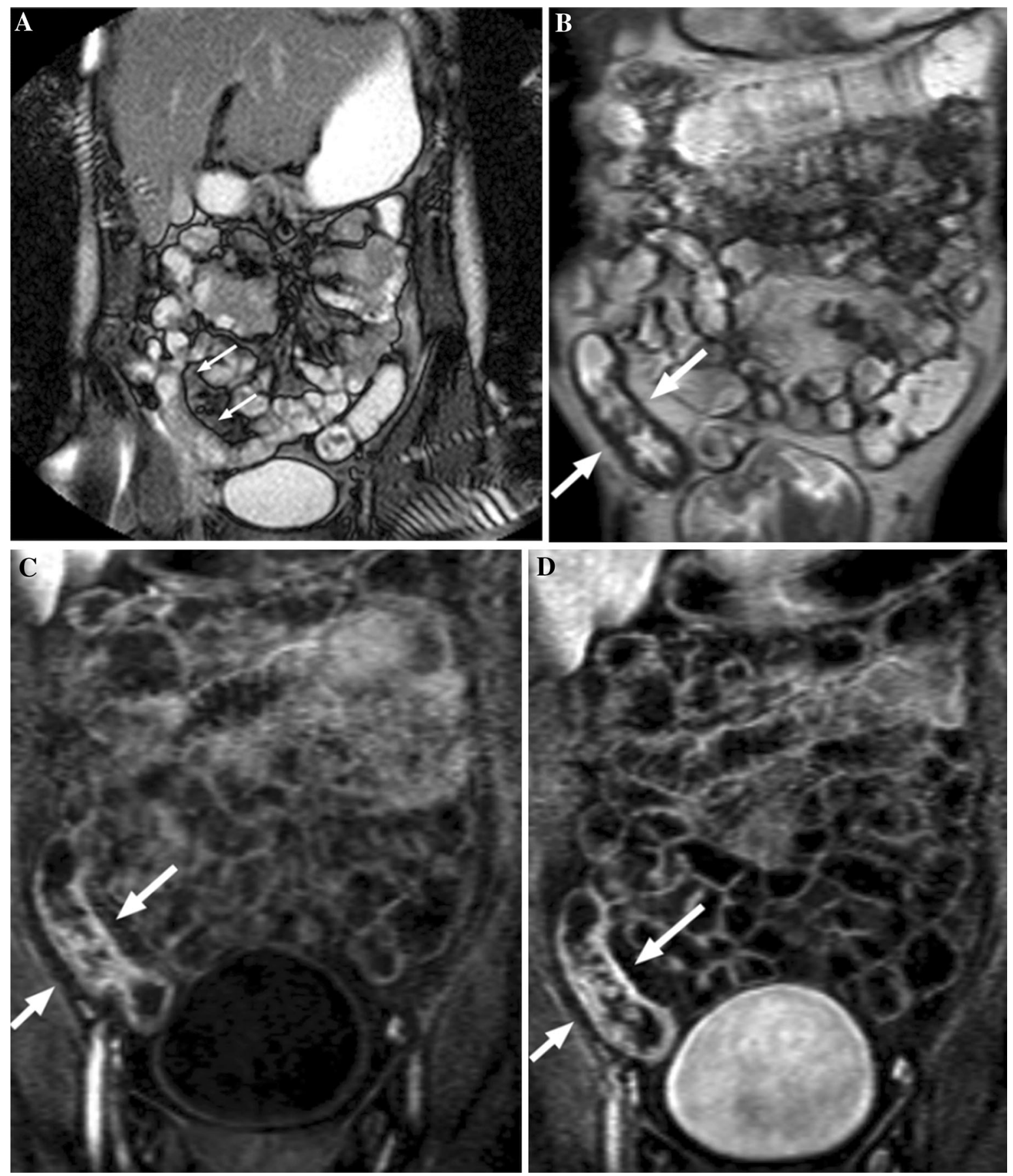

Fig. 3. Active Crohn's disease of the terminal ileum in a 25-year-old woman. A (Video 7)-Cine balanced fast field echo (BFFE) series (TR $3.2 \mathrm{~ms}$, TE $1.6 \mathrm{~ms}$, matrix $160 \times 160$, flip angle $45^{\circ}$, thickness $8 \mathrm{~mm}$ ) shows decreased peristalsis of the abnormal terminal ileum (arrows) compared to the remainder of the small bowel. B Coronal T2-weighted single-shot fast spin echo (FSE) images (TR $\infty$, TE $80 \mathrm{~ms}$, matrix $256 \times 154$, thickness $6 \mathrm{~mm}$ ) showing mild terminal

Germany) which is an agent approved outside of the USA [12]. In the USA, most centers use glucagon (GlucaGen $^{\circledR}$, Novo Nordisk, DK-2880 Bagsvaerd) ileum wall thickening (arrows). C, D 3-D dynamic (C) and delayed post-contrast (D) coronal T1W high-resolution isotropic volume examination (THRIVE) images (TR $4.2 \mathrm{~ms}$, TE $2.1 \mathrm{~ms}$, matrix $256 \times 163$, thickness $5 \mathrm{~mm}$ ) shows mucosal fold thickening with increased mucosal and transmural late arterial enhancement (C) and delayed enhancement (D) with ulceration along the lateral wall (arrows) consistent with active Crohn's disease.

$[26,32]$. However, it is important to note that cine BSSFP sequences should be performed before spasmolytic agents are administered. Otherwise, these agents will 

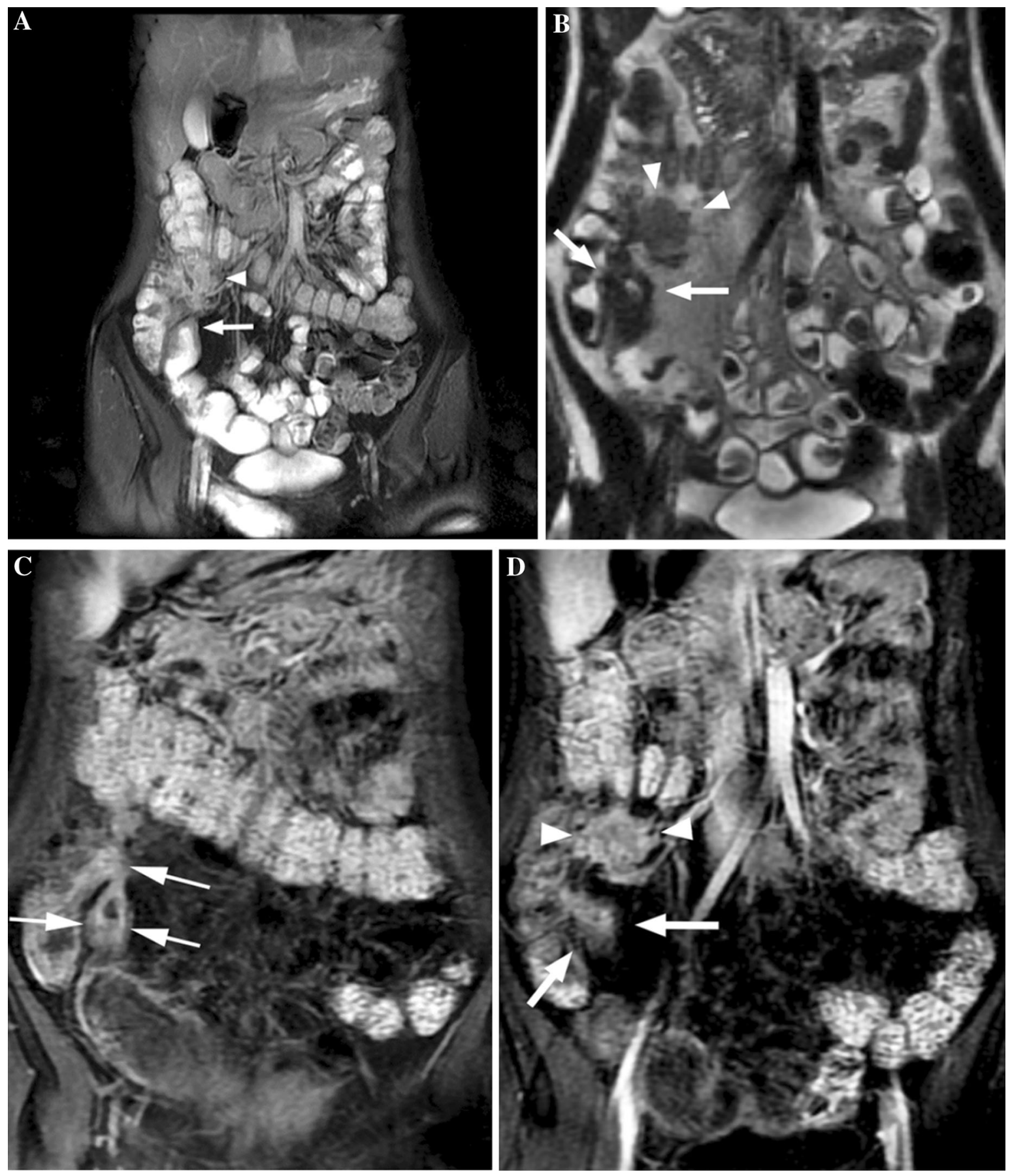

Fig. 4. Active inflammatory and chronic Crohn's disease of the terminal ileum with adjacent phlegmon in a 21-year-old woman. A (Video 8)-Cine fast imaging employing steady-state acquisition (FIESTA) series (TR $3.8 \mathrm{~ms}$, TE $1.7 \mathrm{~ms}$, matrix $224 \times 288$, flip angle $70^{\circ}$, thickness $8 \mathrm{~mm}$ ) shows decreased peristalsis of the abnormal terminal ileum (arrow) compared to the remainder of the small bowel, and adjacent phlegmon (arrowhead). There is upstream small bowel dilatation. B

decrease small bowel peristalsis which will likely limit the accuracy of this sequence in characterizing small bowel peristalsis.

Coronal T2-weighted single-shot fast spin echo (FSE) image (TR $\infty$, TE $181 \mathrm{~ms}$, matrix $256 \times 224$, thickness $5 \mathrm{~mm}$ ) showing wall thickening of the terminal ileum (arrows) and adjacent phlegmon (arrowheads). C, D Dynamic post-contrast coronal liver acquisition with volume acceleration (LAVA) images (TR $4 \mathrm{~ms}$, TE $1.76 \mathrm{~ms}$, matrix $320 \times 224$, thickness $4.4 \mathrm{~mm}$ ) show terminal ileum wall thickening and mucosal hyperenhancement (arrows) and enhancing adjacent phlegmon (arrowheads).

Research has also shown that MRE interpretation may be accurate even without using spasmolytic agents $[6,14,35]$, particularly for evaluating patients with known 

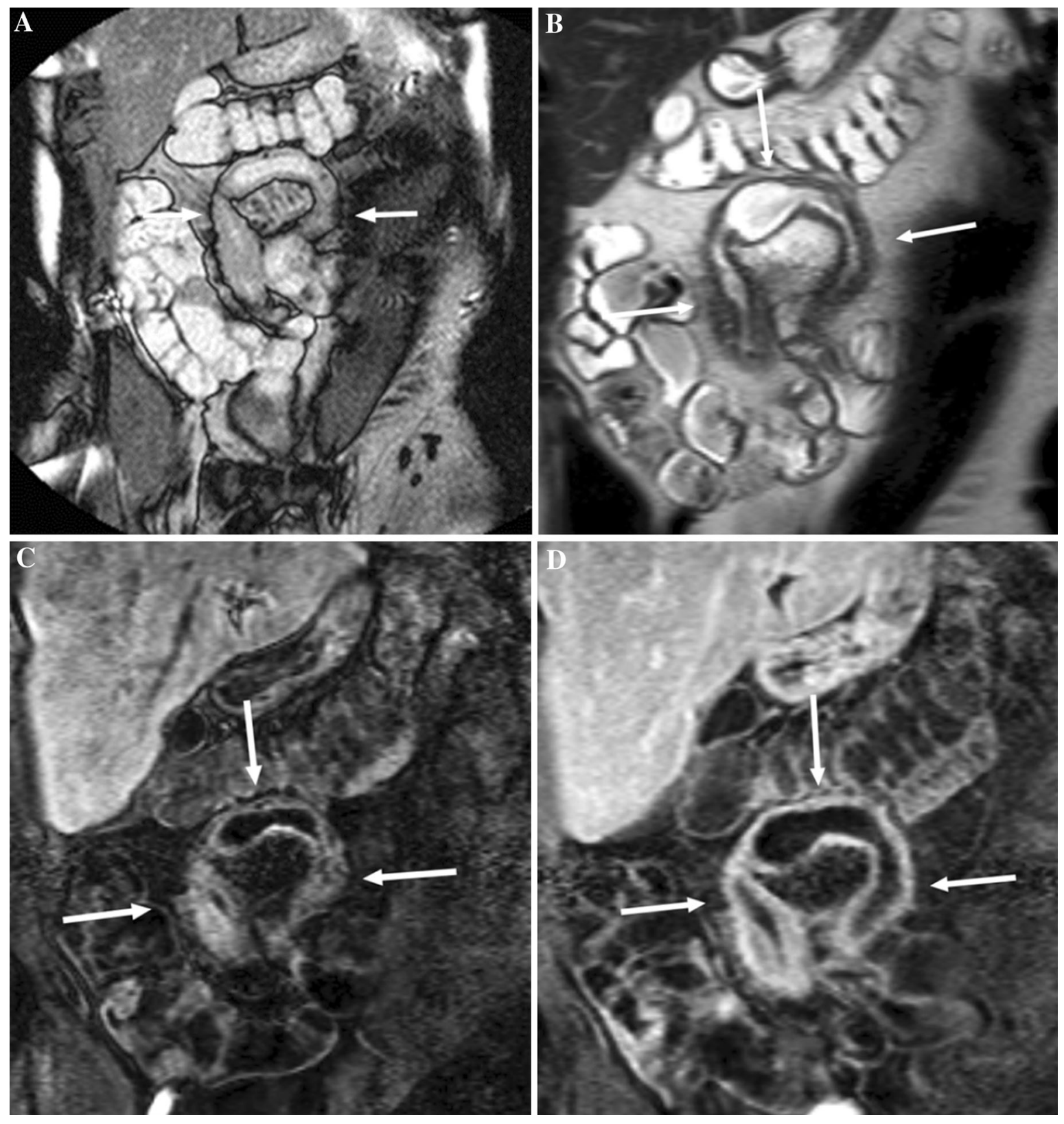

Fig. 5. Active inflammatory and chronic Crohn's disease of the mid ileum in a 66-year-old man. A (Video 9)-Cine balanced fast field echo (BFFE) series (TR $3.2 \mathrm{~ms}$, TE $1.6 \mathrm{~ms}$, matrix $160 \times 160$, flip angle $45^{\circ}$, thickness $8 \mathrm{~mm}$ ) shows decreased peristalsis of the abnormal mid ileum (arrows) compared to the remainder of the small bowel with mild upstream small bowel dilatation. B Coronal T2-weighted singleshot image (TR $\infty$, TE $80 \mathrm{~ms}$, matrix $256 \times 154$, thickness $6 \mathrm{~mm}$ ) shows wall thickening, wall edema, and areas of nar-

or suspected inflammatory bowel disease. Excluding a spasmolytic agent in an MRE protocol shortens exam time, simplifies the exam protocol, reduces exam costs, and eliminates drug side effects, making the exam more tolerable to patients. In fact, eliminating the use of an

rowing with skip lesions in the mid ileum (arrows). C 3-D dynamic post-contrast coronal T1W high-resolution isotropic volume examination (THRIVE) image (TR $4.16 \mathrm{~ms}$, TE $2.1 \mathrm{~ms}$, matrix $256 \times 163$, thickness $5 \mathrm{~mm}$ ) shows wall thickening with mucosal and serosal hyperenhancement causing a stratified enhancement pattern (arrows). D 3-D delayed post-contrast coronal THRIVE image (TR $4.16 \mathrm{~ms}$, TE $2.1 \mathrm{~ms}$, matrix $256 \times 163$, thickness $5 \mathrm{~mm}$ ) shows more homogeneous wall enhancement (arrows).

antiperistaltic agent may actually help diagnose abnormal small bowel segments, based on the difference in peristalsis, effectively becoming a parameter of tissue contrast. The decreased peristalsis of inflamed small bowel segments can decrease the bowel blur that is inherent in $3 \mathrm{D}$ 
Table 2. Sensitivity and specificity of identifying inflamed small bowel segments using the cine BSSFP sequences for diagnosing Crohn's disease for all 5 readers

\begin{tabular}{llllll}
\hline & Reader 1 & Reader 2 & Reader 3 & Reader 4 & Reader 5 \\
\hline Sensitivity & $100 \%(30 / 30)$ & $70 \%(21 / 30)$ & $97 \%(29 / 30)$ & $100 \%(30 / 30)$ & $87 \%(26 / 30)$ \\
Specificity & $100 \%(30 / 30)$ & $93 \%(28 / 30)$ & $100 \%(30 / 30)$ & $100 \%(30 / 30)$ & $97 \%(29 / 30)$ \\
\hline
\end{tabular}

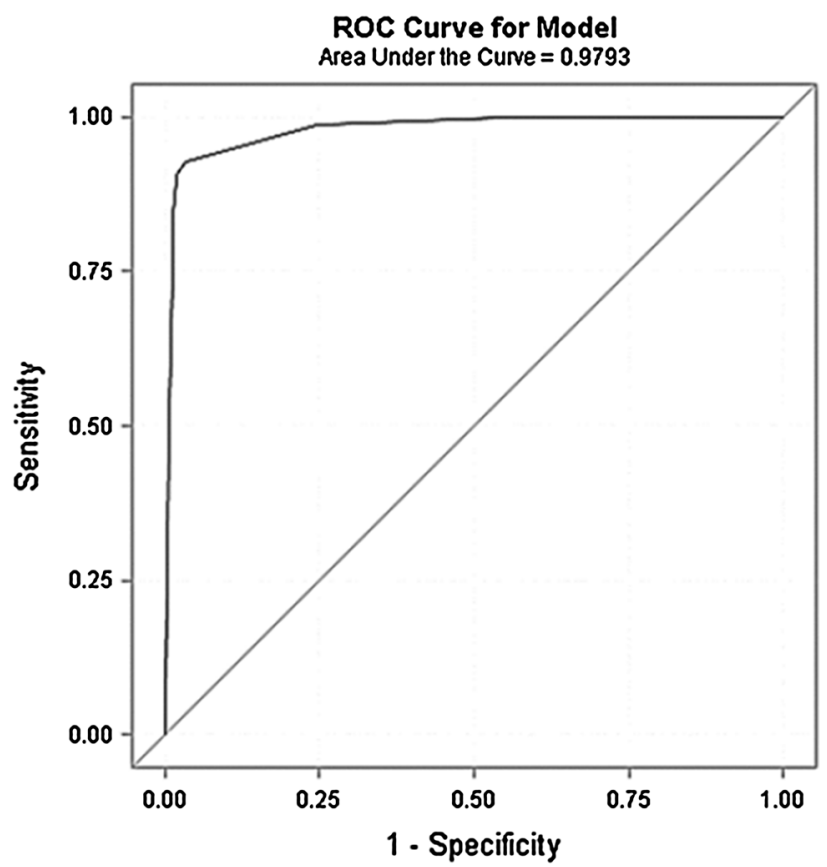

Fig. 6. The combined ROC curve for all 5 readers.

Table 3. Means and standard deviations of overall small bowel peristalsis versus peristalsis of abnormal small bowel segments in Crohn's patients for 5 readers $(n=30)$

\begin{tabular}{llcc}
\hline Reader $^{\mathrm{a}}$ & Variable & Mean & SD \\
\hline 1 & Overall peristalsis & 3.0 & 0.4 \\
& Abnormal segment & 1.0 & 0.0 \\
& Overall peristalsis & 3.0 & 0.7 \\
3 & Abnormal segment & 1.3 & 0.5 \\
& Overall peristalsis & 2.3 & 0.8 \\
4 & Abnormal segment & 1.1 & 0.3 \\
& Overall peristalsis & 3.1 & 0.6 \\
5 & Abnormal segment & 1.2 & 0.4 \\
& Overall peristalsis & 2.7 & 0.8 \\
& Abnormal segment & 1.2 & 0.4 \\
\hline
\end{tabular}

${ }^{a}$ Paired $t$ tests of the peristalsis in abnormal small bowel segments in Crohn's patients with the remainder of the small bowel were significant for all five readers $(t=9.63-26.91, p<.0001)$

GRE sequences, making the abnormal segments stand out compared to normal small bowel [24, 31]. Thus, inflamed small bowel segments may have decreased peristalsis on cine BSSFP sequences and may also be better defined on 3D GRE sequences (Figs. 2, 3, 4, and 5). Since abnormal small bowel segments may appear frozen in time on these sequences, this decreased peristalsis can be termed the "frozen bowel sign."
Table 4. Means and standard deviations of peristalsis in abnormal small bowel segments in Crohn's patients and the overall small bowel peristalsis in normal-MRE patients

\begin{tabular}{llrcc}
\hline Reader $^{\mathrm{a}}$ & CROHNS & $N$ & Mean & SD \\
\hline 1 & No & 30 & 3.1 & 0.7 \\
& Yes & 30 & 1.0 & 0.0 \\
2 & No & 30 & 3.2 & 1.0 \\
& Yes & $21^{\mathrm{b}}$ & 1.3 & 0.5 \\
3 & No & 30 & 2.8 & 0.9 \\
& Yes & $29^{\mathrm{b}}$ & 1.1 & 0.3 \\
4 & No & 30 & 3.5 & 0.9 \\
& Yes & 30 & 1.2 & 0.4 \\
5 & No & 30 & 3.0 & 0.7 \\
& Yes & $26^{\mathrm{b}}$ & 1.2 & 0.4 \\
\hline
\end{tabular}

${ }^{a}$ Independent group $t$ tests of the peristalsis in abnormal small bowel segments in Crohn's patients with the overall small bowel peristalsis in normal-MRE patients are also significant for all readers $(t=9.54$ to $17.38, p<.0001)$

${ }^{\mathrm{b}} N$ is $<30$ for some readers due to less than $100 \%$ accuracy

Table 5. Means and standard deviations of overall small bowel peristalsis in Crohn's patients $(n=30)$ and normal-MRE patients $(n=30)$ for 5 readers

\begin{tabular}{llcl}
\hline Reader & CROHNS & Mean & SD \\
\hline 1 n.s. & No & 3.1 & 0.7 \\
& Yes & 3.0 & 0.4 \\
2 n.s. & No & 3.2 & 1.0 \\
$3^{\text {a }}$ & Yes & 3.0 & 0.7 \\
& No & 2.8 & 0.9 \\
4 n.s. & Yes & 2.3 & 0.8 \\
& No & 3.5 & 0.9 \\
5 n.s. & Yes & 3.1 & 0.6 \\
& No & 3.0 & 0.7 \\
& Yes & 2.7 & 0.8 \\
\hline
\end{tabular}

n.s. not significant

${ }^{\mathrm{a}}$ Independent groups $t$ test $(t=2.14, p<.05)$

In our study, the interobserver reliabilities calculated with the ICC were on the border of poor and fair for evaluating overall small bowel peristalsis (.39), but between very good and excellent for detecting an abnormal small bowel segment (.79). Evaluation of overall small bowel peristalsis is likely more subjective, with five possible peristalsis grades, and might have benefited from further training. However, detecting the presence of an abnormal small bowel segment ("the frozen bowel sign"), which is the more important measure, had more than acceptable reliability.

This study has several limitations. Recent pathologic proof was not available for 14 of the 30 abnormal cases evaluated in the study. However, each of the 30 patients with abnormal MRE exams had an established clinical diagnosis of Crohn's disease and all patients had a 
biopsy confirming Crohn's and/or bowel inflammation. Also, obtaining pathologic proof confirming bowel inflammation is one of the limitations of MRE research in general. In most cases, pathologic proof in the small bowel is only available for the terminal ileum, which is the small bowel segment most accessible by colonoscopy, or by surgical resection. Cases that have surgical resection will by definition have more advanced disease that does not respond to more conservative treatment, thus skewing results.

Another study limitation is that not all patients were scanned in the prone position (85\% of patients) which may affect the overall peristalsis rate due to differences in patient positioning, while the cine acquisition was variable at $1-2$ s per image. Both of these factors may have decreased the accuracy of overall small bowel peristalsis evaluation between different patients. However, this will likely have little effect when evaluating the peristalsis of an abnormal small bowel segment compared to the overall small bowel for the same patient. Another limitation is that this study is not a prospective study with random assignment, and therefore selection bias is possible at the different stages of the study design. Next, decreased small bowel peristalsis is not specific for Crohn's disease and can be due to a number of conditions including adhesions, motility disorders, or other segmental or diffuse small bowel processes. Finally, we did not evaluate if peristalsis was affected differently in active vs. chronically inflamed small bowel segments or in active bowel inflammation that involves only the small bowel mucosa vs. the bowel wall.

In conclusion, abnormal small bowel segments in Crohn's disease have significantly decreased peristalsis compared to normal small bowel segments in both Crohn's disease patients and patients without a history of inflammatory bowel disease. The cine BSSFP series is excellent for recognizing this decreased peristalsis, making it a valuable addition to the standard MRE protocol.

\section{References}

1. Booya F, Fletcher JG, Huprich JE, et al. (2006) Active crohn disease: CT findings and interobserver agreement for enteric phase CT enterography. Radiology 241(3):787-795.

2. Bodily KD, Fletcher JG, Solem CA, et al. (2006) Crohn disease: mural attenuation and thickness at contrast-enhanced CT enterography - correlation with endoscopic and histologic findings of inflammation. Radiology 238(2):505-516.

3. Choi D, Jin LS, Ah CY, et al. (2003) Bowel wall thickening in patients with Crohn's disease: CT patterns and correlation with inflammatory activity. Clin Radiol 58(1):68-74.

4. Maglinte DDT, Gourtsoyiannis N, Rex D, et al. (2003) Classification of small bowel Crohn's subtypes based on multimodality imaging. Radiol Clin North Am 41(2):285-304.

5. Florie J, Wasser MNJM, Arts-Cieslik K, et al. (2006) Dynamic contrast-enhanced MRI of the bowel wall for assessment of disease activity in Crohn's disease. Am J Roentgenol 186(5):1384-1392.

6. Siddiki HA, Fidler JL, Fletcher JG, et al. (2009) Prospective comparison of state-of-the-art MR enterography and CT enterography in small-bowel Crohn's disease. Am J Roentgenol 193(1):113-121.
7. Rimola J, Rodríguez S, García-Bosch O, et al. (2009) Magnetic resonance for assessment of disease activity and severity in ileocolonic Crohn's disease. Gut 58(8):1113-1120.

8. Messaris E, Chandolias N, Grand D, et al. (2010) Role of magnetic resonance enterography in the management of Crohn disease. Arch Surg 145(5):471-475.

9. Punwani S, Rodriguez-Justo M, Bainbridge A, et al. (2009) Mural inflammation in crohn disease: location-matched histologic validation of MR imaging features. Radiology 252(3):712-720.

10. Sinha R, Rajiah P, Murphy P, et al. (2009) Utility of high-resolution MR imaging in demonstrating transmural pathologic changes in Crohn disease. Radiographics 29(6):1847-1867.

11. Steward MJ, Punwani S, Proctor I, et al. (2011) Non-perforating small bowel Crohn's disease assessed by MRI enterography: Derivation and histopathological validation of an MR-based activity index. Eur J Radiol 81(9):2080-2088.

12. Ziech M, Bossuyt P, Laghi A, et al. (2011) Grading luminal Crohn's disease: Which MRI features are considered as important? Eur J Radiol 81(4):e467-e472.

13. Ziech MLW, Bipat S, Roelofs JJTH, et al. (2011) Retrospective comparison of magnetic resonance imaging features and histopathology in Crohn's disease patients. Eur J Radiol 80(3):e299-e305.

14. Grand DJ, Kampalath V, Harris A, et al. (2012) MR enterography correlates highly with colonoscopy and histology for both distal ileal and colonic Crohn's disease in 310 patients. Eur J Radiol 81(5):e763-e769.

15. Sempere GAJ, Sanjuan VM, Chulia EM, et al. (2005) MRI evaluation of inflammatory activity in Crohn's disease. Am J Roentgenol 184(6):1829-1835.

16. Oto A, Zhu F, Kulkarni K, et al. (2009) Evaluation of diffusionweighted MR imaging for detection of bowel inflammation in patients with Crohn's disease. Acad Radiol 16(5):597-603.

17. Oto A, Kayhan A, Williams JTB, et al. (2011) Active Crohn's disease in the small bowel: evaluation by diffusion weighted imaging and quantitative dynamic contrast enhanced MR imaging. J Magn Reson Imaging 33(3):615-624.

18. Oussalah A, Laurent V, Bruot O, et al. (2010) Diffusion-weighted magnetic resonance without bowel preparation for detecting colonic inflammation in inflammatory bowel disease. Gut 59(8):10561065.

19. Kiryu S, Dodanuki K, Takao H, et al. (2009) Free-breathing diffusion-weighted imaging for the assessment of inflammatory activity in Crohn's disease. J Magn Reson Imaging 29(4):880-886.

20. Ghobrial PM, Neuberger I, Guglielmo FF, et al. (2014) Cine MR enterography grading of small bowel peristalsis. evaluation of the antiperistaltic effectiveness of sublingual hyoscyamine sulfate. Acad Radiol 21(1):86-91.

21. Wakamiya M, Furukawa A, Kanasaki S, et al. (2011) Assessment of small bowel motility function With cine-MRI using balanced steady-state free precession sequence. J Magn Reson Imaging 33(5):1235-1240.

22. Patak MA, Froehlich JM, Von Weymarn C, et al. (2007) Noninvasive measurement of small-bowel motility by MRI after abdominal surgery. Gut 56(7):1023-1025.

23. Froehlich JM, Patak MA, von Weymarn C, et al. (2005) Small bowel motility assessment with magnetic resonance imaging. J Magn Reson Imaging 21(4):370-375.

24. Odille F, Menys A, Ahmed A et al. (2012) Quantitative assessment of small bowel motility by nonrigid registration of dynamic MR images. Magn Reson Med 68(3):783-793.

25. Heye T, Stein D, Antolovic D, et al. (2012) Evaluation of bowel peristalsis by dynamic cine MRI: Detection of relevant functional disturbances - initial experience. J Magn Reson Imaging 35(4):859867.

26. Gutzeit A, Binkert CA, Koh DM et al. (2012) Evaluation of the anti-peristaltic effect of glucagon and hyoscine on the small bowel: comparison of intravenous and intramuscular drug administration. Eur Radiol 22(6):1186-1194.

27. Kitazume Y, Satoh S, Hosoi H, et al. (2007) Cine magnetic resonance imaging evaluation of peristalsis of small bowel with longitudinal ulcer in Crohn disease: preliminary results. J Comput Assist Tomogr 31(6):876-883.

28. Menys A, Atkinson D, Odille F et al. (2012) Quantified terminal ileal motility during MR enterography as a potential biomarker of 
Crohn's disease activity: a preliminary study. Eur Radiol 22(11): 2494-2501.

29. Bickelhaupt S, Pazahr S, Chuck N et al. Crohn's disease: small bowel motility impairment correlates with inflammatory-related markers C-reactive protein and calprotectin. Neurogastroenterol Motil 2013;25(6):467-e363.

30. Girometti R, Zuiani C, Toso F, et al. (2008) MRI scoring system including dynamic motility evaluation in assessing the activity of Crohn's disease of the terminal ileum. Acad Radiol 15(2):153-164.

31. Froehlich JM, Waldherr C, Stoupis C, et al. (2010) MR motility imaging in Crohn's disease improves lesion detection compared with standard MR imaging. Eur Radiol 20(8):1945-1951.
32. Froehlich JM, Daenzer M, von Weymarn C, et al. (2009) Aperistaltic effect of hyoscine $N$-butylbromide versus glucagon on the small bowel assessed by magnetic resonance imaging. Eur Radiol 19(6):1387-1393.

33. Shrout PE, Fleiss JL (1979) Intraclass correlations: uses in assessing rater reliability. Psychol Bull 86(2):420.

34. Koh D, Miao Y, Chinn R, et al. (2001) MR imaging evaluation of the activity of Crohn's disease. Am J Roentgenol 177(6):1325-1332

35. Grand DJ, Beland MD, Machan JT et al. Detection of Crohn's disease: Comparison of CT and MR enterography without antiperistaltic agents performed on the same day. Eur J Radiol 2011; 81(8):1735-1741. 